
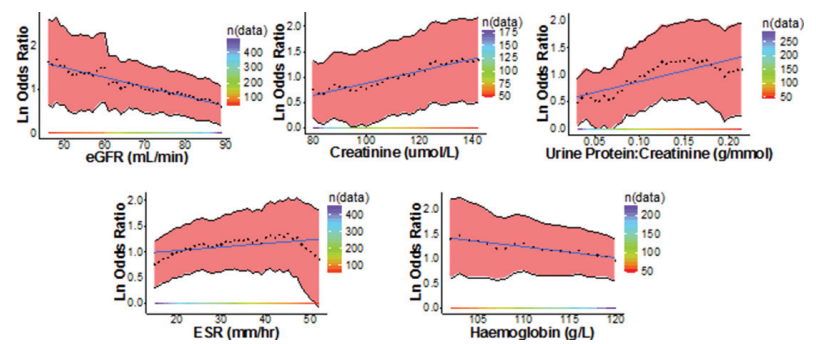

Abstract FRI0359 - Figure 1. Ln (natural log) odds ratio for damage transition vs timeadjusted mean of laboratory variables. Red region $(95 \% \mathrm{Cl}$ ) lying above the $\mathrm{y}=0$ line indicates the risk is statically significant $(\mathrm{p}<0.05)$. Regression lines (blue) suggest the risk of damage increase is approximately proportional with the distance from normal pathology measure range.

Conclusions: Routine pathology measures were found to be proportionally associated with organ damage risk in SLE. The potential for the use of these measures as biomarkers, for example to generate an organ damage risk calculator for SLE, warrants further research.

Disclosure of Interest: None declared

DOI: 10.1136/annrheumdis-2018-eular.5226

\section{FRI0360 DISEASE SEVERITY OF PROLIFERATIVE LUPUS NEPHRITIS IN MAGHREBIANS}

F. Tamirou ${ }^{1}$, N. Costedoat-Chalumeau ${ }^{2}$, G. Medkouri $^{3}$, E. Daugas ${ }^{4}$, E. Hachulla ${ }^{5}$, N. Jourde-Chiche ${ }^{6}$, A. Karras ${ }^{7}$, V. le Guern ${ }^{8}$, V. Gnemmi ${ }^{9}$, M. Jadoul $^{10}$, F. A. Houssiau'. ${ }^{1}$ Rheumatology Department, Cliniques Universitaires Saint-Luc, Université catholique de Louvain, Brussels, Belgium, ${ }^{2}$ Internal Medicine Department, Centre de référence des maladies auto-immunes et systémiques rares d'île de France, AP-HP, Hôpital Cochin, Université Descartes-Sorbonne, Paris, France; ${ }^{3}$ Nephrology Department, Centre Hospitalier Universitaire Ibn Rochd, Casablanca, Morocco; ${ }^{4}$ Nephrology Department, AP-HP, Hôpital Bichat, Université Paris Diderot, Paris; ${ }^{5}$ Internal Medicine Department, Hôpital Claude Huriez, Université Lille Nord, Lille; ${ }^{6}$ Nephrology Department, AP-HM, Hôpital de la Conception, Aix-Marseille Université, Marseille; ${ }^{7}$ Nephrology Department, AP-HP, Hôpital Européen Georges-Pompidou, Université Paris Descartes; ${ }^{8}$ Internal Medicine Department, Centre de référence des maladies auto-immunes et systémiques rares d'île de France, AP-HP, Hôpital Cochin, Université Paris Descartes-Sorbonne, Paris; ${ }^{9}$ Pathology Institute, Université Lille Nord, Lille, France; ${ }^{10}$ Nephrology Department, Cliniques Universitaires Saint-Luc, Université catholique de Louvain, Brussels, Belgium

Background: The negative influence of African-American ethnicity on the prognosis of lupus nephritis $(\mathrm{LN})$ is well known but - to the best of our knowledge - the impact of Maghrebian ethnicity has never been evaluated, although the disease is purported to be quite prevalent and severe in North Africa.

Objectives: To study the influence of Maghrebian ethnicity on the clinical and pathological presentation of LN, the renal relapse rate, the renal and overall survival and the predictive value of an early proteinuria decrease for good long-term renal outcome in this population compared to native Europeans.

Methods: We retrospectively reviewed the files of an inception cohort of 194 patients with proliferative LN followed in 7 lupus centres belonging to three groups: Europeans living in Belgium/France $(E ; n=111)$, Maghrebians living in Europe, in casu Belgium/France (ME; $n=43$ ) and Maghrebians living in Morocco $(\mathrm{MM} ; \mathrm{n}=40)$. Baseline presentation was compared between these 3 groups but complete long-term outcome data were available only for $E$ and $M E$ patients.

Results: At presentation, clinical (gender, age, nephrotic syndrome, serum creatinine, eGFR, uP/C ratio) and pathological (ISN/RPS class) characteristics of LN did not differ between $E, M E$ and $M M$ patients. At one year, renal remission was met in $73 \%, 63 \%$ and $68 \%$ in E, ME and MM patients, respectively. Achievement of a target proteinuria below $0.7 \mathrm{~g} /$ day one year after treatment initiation was $76 \%$, $63 \%$ and $68 \%$, respectively. Nevertheless, while proteinuria measured at month 12 accurately predicted a serum creatinine value $<1 \mathrm{mg} / \mathrm{dl}$ at 7 years in $E$ patients, this was not the case in the ME group, in whom serum creatinine at month 12 performed better. Renal relapses were more common in ME patients (54\%) than in $\mathrm{E}$ patients $(29 \%)(p<0.01)$. Time to renal flare and to ESRD was shorter in ME patients compared to $E$ patients $(p<0.0001$ and $p<0.05$, respectively) as shown in figure 1. At last follow-up, mean proteinuria, serum creatinine and eGFR did not differ between $E$ and $M E$ patients, nor did the percentage of patients who died or suffered from ESRD or permanent renal impairment.

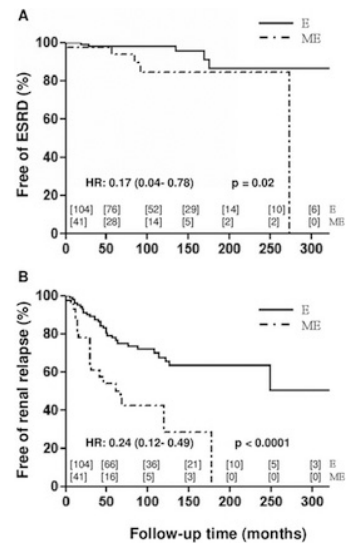

Conclusions: Despite a similar disease profile at onset, the prognosis of $L N$ is more severe in Maghrebians living in Europe compared to native Europeans, with a higher relapse rate and a shorter time to ESRD.

Disclosure of Interest: None declared

DOI: 10.1136/annrheumdis-2018-eular.3070

\section{FRI0361 PREGNANCY OUTCOMES AND THERAPY IN SYSTEMIC LUPUS ERYTHEMATOSUS: RESULTS FROM OUR 30 YEARS' EXPERIENCE PREGNANCY CLINIC}

F. Crisafullii ${ }^{1}$, M.G. Lazzaroni ${ }^{1}$, L. Andreoli ${ }^{1}$, M.C. Gerardi ${ }^{1}$, L. Antolini ${ }^{1}$, G. Parma ${ }^{1}$, C. Benigno ${ }^{2}$, A. Lojacono ${ }^{3}$, S. Zatti ${ }^{3}$, V. Cappa ${ }^{4}$, S. Calza ${ }^{4}$, F. Franceschini ${ }^{1}$, A. Tincani'. ' U.O. Rheumatology and Clinical Immunology, Spedali Civili and University of Brescia, Brescia; ${ }^{2}$ U.O. Rheumatology and Clinical Immunology, Federico II University Hospital and University of Napoli, Napoli; ${ }^{3}$ Obstetrics and Gynecology Unit, ${ }^{4}$ Biostatistics and Biomathematics Unit and Bioinformatics Unit, Spedali Civili and University of Brescia, Brescia, Italy

Background: The outcome of Systemic Lupus Erythematosus(SLE) pregnancies has dramatically improved thanks to pregnancy planning, multidisciplinary management and close monitoring during pregnancy. In our experience, programmed pregnancies in SLE patients had similar rates of pregnancy losses as compared to general obstetric population, but there are still open issues on some pregnancy complications that more frequently affect SLE patients.

Objectives: To analyse the obstetric outcome of SLE patients, according to specific therapy received during pregnancy.

Methods: A monocentric, retrospective study of 98 SLE patients with a total of 134 pregnancies followed prospectively by multidisciplinary team(1987-2015). Adverse Pregnancy Outcomes(APOs) were defined as one of the followings:premature miscarriage ( $<10$ th week), intrauterine fetal death $(>10$ th week), perinatal death ( $<30$ th day of life), severe preterm birth $(<34$ th week) and preterm birth (between 34th-36th weeks). We also evaluated the frequency of other pregnancy complications such as preterm premature rupture of membranes(PPROM) and pre-eclampsia(PE).

Results: Among the 134 pregnancies(including 3 twin pregnancies), flares occurred in $10(7.5 \%)$ and APOs in $39(29.1 \%)$ cases (table 1). pPROM complicated 9 pregnancies, all resulted in preterm deliveries, including 3 severe preterm birth; PE complicated 6 pregnancies resulting in 2 preterm birth, 1 intrauterine fetal death, 1 perinatal death and 2 term birth. The rates of APOs, pPROM and PE were compared according to receiving or not a specific therapy: hydroxychloroquine(HCQ), low dose aspirin(LDA), immunosuppressant(IS) during the overall pregnancy and corticosteroids $>35 \mathrm{mg} / \mathrm{week}$ (CS) during the $1 \mathrm{st}$, the $2 \mathrm{nd}$ and the 3rd trimester. No statistical significant association was found between a specific therapy and the rate of PE. HCQ and LDA did not significantly affected the rate of APOs or pPROM while pregnancies exposed to IS showed a higher frequency of APOs $(47 \%$ vs $20 \%, p 0.003)$, in particular premature miscarriages $(16 \%$ vs $2 \%$, p0.007). Pregnancies exposed to CS had higher frequency of APOs (1 st trimester $44 \%$ vs $28 \%$, p0.015; 2nd trimester $36 \%$ vs $13 \%$ p0.004; 3rd trimester $34 \%$ vs $14 \%, p 0.019)$. Considering only the 120 pregnancies resulted in live birth, those exposed to CS had higher frequency of preterm birth (1 st trimester p0.006; 2nd trimester p0.023; 3rd trimester p0.011) (table 2). In particular, pregnancies exposed to CS in the 1 st trimester had higher frequency of preterm birth on 34th-36th w $(p 0.017)$, while pregnancies exposed in the 3rd trimester had a higher frequency of preterm birth before 34 th $w(p 0.038)$. Furthermore, a higher frequency of pPROM was observed in those exposed to CS $(1$ st trimester $00.001 ; 2$ nd trimester p0.003; 3rd trimester p0.001). 
Abstract FRI0361 - Table 1. frequency of APOs, pPROM and PE in the 134 analysed pregnancies

\begin{tabular}{|l|l|}
\cline { 2 - 2 } \multicolumn{1}{c|}{} & \multicolumn{1}{c|}{$\mathbf{N}(\%)$} \\
\hline N of live birth & $\mathbf{1 2 0 ( 9 0 \% )}$ \\
\hline N of APOs & $39(29 \%)$ \\
\hline Premature miscarriage $\left(<10^{\text {th }}\right.$ w) & $\mathbf{9 ( 7 \% )}$ \\
\hline Intrauterine fetal death $\left(>10^{\text {th }}\right.$ w) & $5(4 \%)$ \\
\hline with PE & $1(20 \%)$ \\
\hline Perinatal death $\left(<30^{\text {th }}\right.$ day of life) & $\mathbf{1 ( 1 \% )}$ \\
\hline with PE & $1(100 \%)$ \\
\hline All preterm birth $\left(<37^{\text {th }}\right.$ w) & $\mathbf{2 4 ( 1 8 \% )}$ \\
\hline Preterm birth $\left(<34^{\text {th }}\right.$ w) & $\mathbf{3 ( 2 \% )}$ \\
\hline with $p P R O M$ & $2(67 \%)$ \\
\hline Preterm birth $\left(34^{\text {th }}-36^{\text {th }}\right.$ w) & $\mathbf{2 1 ( 1 6 \% )}$ \\
\hline with $p P R O M$ & $7(33 \%)$ \\
\hline with PE & $2(10 \%)$ \\
\hline
\end{tabular}

Abstract FRI0361 - Table 2. frequency of preterm birth and pPROM among the 120 live birth, according to exposure (or not) to corticosteroids $>35 \mathrm{mg} /$ week during the three trimesters

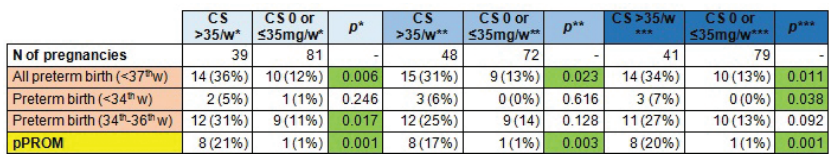

* 1 st trimester; ** 2nd trimester; *** 3 rd trimester

Conclusions: Use of IS seems to be associated with premature miscarriages; this could reflect their use in patients with a more severe disease phenotype. The exposure to CS in doses greater than $35 \mathrm{mg} / \mathrm{w}$ in the $1 \mathrm{st}$ trimester seems to be associated with preterm birth at 34th-36th w, while in the 3rd trimester with severe preterm birth $(<34$ th $w)$, that could be related to the strong association observed between CS use and pPROM.

Disclosure of Interest: None declared

DOI: 10.1136/annrheumdis-2018-eular.5085

\section{FRI0362 PREVALENCE, RISK FACTORS, AND IMPACT ON MORTALITY OF NEUROPSYCHIATRIC LUPUS: A LARGE PROSPECTIVE SINGLE-CENTRE STUDY}

G.Y. Ahn ${ }^{1}$, D. Kim ${ }^{1}$, S. Won ${ }^{2}$, S.T. Song ${ }^{1}$, H.-J. Jeong ${ }^{1}$, I.W. Sohn ${ }^{1}$, S. Lee ${ }^{1}$, Y B. Joo ${ }^{3}$, S.-C. Bae ${ }^{1}{ }^{1}$ Hanyang University Hospital for Rheumatic Diseases; ${ }^{2}$ Clinical Research Center for Rheumatoid Arthritis (CRCRA), Seoul; ${ }^{3}$ St. Vincent's Hospital, The Catholic University of Korea, Suwon, Korea, Republic Of

Background: Neuropsychiatric involvement is one of the most serious involvement of SLE and generally associated with a worse prognosis. However, previous reports about the prevalence and risk factors of neuropsychiatric systemic lupuc erythematosus (NPSLE) have yielded inconsistent findings. Also, there are only few studies of the prognosis of NPSLE, especially in a large prospective cohort. Objectives: To identify the prevalence, risk profiles, and impact on mortality of NPSLE.

Methods: Patients from the Hanyang BAE lupus cohort were registered and followed from 1998 to 2015. Demographics, autoantibodies, SLEDAI-2K and SLICC/ACR damage index were collected at baseline and then annually. Patients registered within 12 months of SLE development were grouped as the inception cohort and analysed separately to elucidate the clinical features at disease onset. NP manifestations were defined using the ACR 19 case definitions and Ainiala NPSLE that did not include minor NP events ${ }^{1}$. Mortality data were derived by linking data from the Korean National Statistics Office.

Results: The prevalence of NPSLE by ACR 19 case definition was $38.3 \%$, and $19.3 \%$ by Ainiala criteria. Higher SLEDAI, APLA positivity, absence of anti-dsDNA antibody at SLE diagnosis and fewer years of education incresed NPSLE risk.
Patients with any NPSLE manifestation had a three-fold increased risk of mortality (HR 3.09, $p=0.04$ ), and patients with focal CNS NPSLE showed nearly an eightfold increased risk of mortality in SLE patients (HR=7.83, $p<0.01)$. Among the 216 patients with Ainiala NPSLE, sixty-four (29.6\%) had multiple events. The two most common symptom combinations were seizure with CVA (18 patients) and seizure with psychosis (8 patients).

Abstract FRI0362 - Table 1. Risk factors for the development of NPSLE in the inception cohort $(\mathrm{N}=388)$

\begin{tabular}{|c|c|c|c|c|c|}
\hline & $\begin{array}{l}\text { NPSLE } \\
(\mathrm{n}=88)\end{array}$ & $\begin{array}{l}\text { Non-NPSLE } \\
(n=300)\end{array}$ & $P$ & $\begin{array}{l}\text { Adjusted OR } \\
(95 \% \mathrm{Cl})\end{array}$ & $P$ \\
\hline \multicolumn{6}{|l|}{ Demographics } \\
\hline Age at SLE diagnosis & $\begin{array}{r}28.2 \\
\pm 11.6\end{array}$ & $29.3 \pm 11.3$ & 0.42 & $\begin{array}{c}0.99(0.97- \\
1.01)\end{array}$ & 0.45 \\
\hline Female sex & $\begin{array}{c}81 \\
(92.1)\end{array}$ & $270(90.0)$ & 0.71 & $\begin{array}{c}1.88(0.71- \\
4.96)\end{array}$ & 0.20 \\
\hline $\begin{array}{l}\text { SLEDAI at diagnosis, NP } \\
\text { manifestations excluded }^{\text {a }}\end{array}$ & $\begin{array}{l}4.7 \\
\pm 4.5\end{array}$ & $3.8 \pm 3.3$ & 0.10 & $\begin{array}{l}1.08(1.01- \\
1.16)\end{array}$ & $0.02^{*}$ \\
\hline $\begin{array}{l}\mathrm{SDI} \text { at diagnosis, NP } \\
\text { manifestations excluded }^{\mathrm{b}}\end{array}$ & $\begin{array}{c}0.2 \\
\pm 0.4\end{array}$ & $0.1 \pm 0.4$ & 0.43 & $\begin{array}{c}1.23(0.72- \\
2.11)\end{array}$ & 0.45 \\
\hline HTN & $5(5.8)$ & $16(5.4)$ & 1.00 & $\begin{array}{c}1.14(0.38- \\
3.37)\end{array}$ & 0.82 \\
\hline Education (years) & $\begin{array}{l}12.2 \\
\pm 3.1\end{array}$ & $13.0 \pm 3.3$ & $0.05^{*}$ & $\begin{array}{l}0.92(0.85- \\
1.00)\end{array}$ & $0.04^{*}$ \\
\hline Anti-dsDNA antibody positivity & $\begin{array}{c}61 \\
(69.3)\end{array}$ & $238(79.3)$ & 0.07 & $\begin{array}{c}0.43(0.24- \\
0.78)\end{array}$ & $<0.01^{*}$ \\
\hline APLA positivity & $\begin{array}{c}40 \\
(45.5)\end{array}$ & $96(32.0)$ & $0.03^{*}$ & $\begin{array}{c}1.72(1.03- \\
2.87)\end{array}$ & $0.04^{*}$ \\
\hline
\end{tabular}

*Values are number (percentage) or mean \pm standard deviation. a. SLEDAI was calculated using the remaining items except CVA, seizure, organic brain syndrome, psychosis, visual disturbance, cranial nerve disorder. b.SDI was calculated using the remaining items except CVA, myelopathy, seizure, psychosis, cognitive impairment, carinal neuropathy, and peripheral neuropathy.
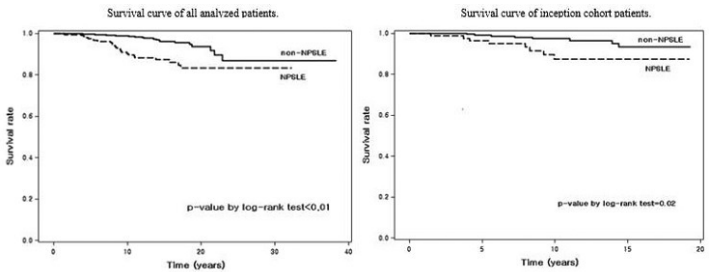

Abstract FRI0362 - Figure 1. Kaplan-Meier survival curves for NPSLE and non-NPSLE patients; Inception cohort and all analysed patients.

Conclusions: Higher SLEDAI, APLA positivity, absence of anti-dsDNA antibody at SLE diagnosis and fewer years of education are risk factors for development of NPSLE. Presence of NPSLE, especially focal CNS NPSLE, increased the risk of mortality in SLE patients.

\section{REFERENCE:}

[1] Ainiala H, Hietaharju A, Loukkola J, et al. Validity of the new American College of Rheumatology criteria for neuropsychiatric lupus syndromes: a population-based evaluation. Arthritis Rheum. 2001;45:419-423.

Disclosure of Interest: None declared

DOI: 10.1136/annrheumdis-2018-eular.4058

\section{FRI0363 POSITIVE REMODELLING INDEX AND LOW ATTENUATION NON-CALCIFIED CORONARY PLAQUES: MARKERS OF VULNERABLE CORONARY PLAQUES IN SYSTEMIC LUPUS?}

G. Stojan ${ }^{1}$, L. Magder ${ }^{2}$, M. Petri ${ }^{1} .{ }^{1}$ Rheumatology, Johns Hopkins University; ${ }^{2}$ Epidemiology, University of Maryland, Baltimore, USA

Background: Accelerated atherosclerosis leading to premature coronary artery disease remains the major cause of late death in SLE. Coronary plaques with large necrotic/lipid core and/or a thin fibrous cap are prone to rupture, leading to acute coronary events. In coronary CT angiography, plaque lipid content correlates with lower CT attenuation values when compared with fibrotic tissue 